\title{
The influence of wearing schemes and supportive telephone calls on adherence in accelerometry measurement: results of a randomized controlled trial
}

\author{
This article was published in the following Dove Press journal: \\ Patient Preference and Adherence \\ 22 March 2017 \\ Number of times this article has been viewed
}

\author{
Neeltje van den Berg ${ }^{1,2}$ \\ Sabina Ulbricht ${ }^{2,3}$ \\ Thea Schwaneberg', \\ Kerstin Weitmann ${ }^{1,2}$ \\ Franziska Weymar ${ }^{1-3}$ \\ Stefan Groß $\beta^{2,4}$ \\ Marcus Dörr ${ }^{2,4}$ \\ Wolfgang Hoffmann ${ }^{1,2}$ \\ 'Institute for Community \\ Medicine, University Medicine \\ Greifswald, ${ }^{2}$ German Centre for \\ Cardiovascular Research (DZHK), \\ Partner Site Greifswald, ${ }^{3}$ Institute \\ of Social Medicine and Prevention, \\ University Medicine Greifswald, \\ ${ }^{4}$ Department of Internal Medicine B, \\ University Medicine Greifswald, \\ Greifswald, Germany
}

Background: Physical activity (PA) can be assessed by accelerometer monitors. However, a high adherence to wearing this device is essential to obtain valid data. In this study, the influence of different wearing schemes and additional supportive phone calls (SPCs) on adherence was examined.

Methods: A randomized study with four groups was conducted in the context of a health examination program among participants aged 40-75 years without a history of cardiovascular diseases. Participants were recruited in different settings (general medical practices, job center, and health insurance). The participants were asked to wear an accelerometer for 7 consecutive days according to the wearing scheme "day and night" or "day only" and received or did not receive SPCs. Full adherence was defined as a total wearing time of 98 hours (between 8 am and $10 \mathrm{pm}$ during 7 days). A generalized linear model was used to calculate the difference between the maximum possible and the observed adherence.

Results: Adherence could be assessed for 249 participants (mean age: 56.40 years; standard deviation [SD] 9.83, 40\% males). The mean wearing time was 84.04 hours (SD 20.75). Participants with the wearing scheme day and night were significantly more adherent than participants with the wearing scheme day only (incidence rate ratio [IRR] $0.63 ; P=0.005$ ). SPCs had no additional effect on adherence (IRR $0.80 ; P=0.168$ ).

Conclusion: To assess PA, the wearing scheme day and night provides the best possible adherence in this group of participants. Further studies are necessary to examine adherence and the effects of additional SPCs in other samples or settings.

Keywords: accelerometry, physical activity assessment, health promotion

\section{Introduction}

Physical activity (PA) is defined as any body movements resulting in energy expenditure. ${ }^{1}$ Various studies indicate positive effects of moderate and vigorous PA on survival $^{2}$ and an association with a reduced risk of cardiovascular diseases. ${ }^{3-5}$ A metaanalysis comparing drug therapy with exercise suggested similar effects on mortality with respect to secondary prevention of coronary heart diseases. ${ }^{5}$ Consequently, PA is an important target being addressed in current prevention strategies, which aims to reduce the risk of cardiovascular disease. ${ }^{6,7}$

PA can be measured directly by, eg, steps, counts, metabolic equivalents of tasks, frequency, and intensity using an accelerometer device, whereas a self-report as an
Correspondence: Neeltje van den Berg Institute for Community Medicine, University Medicine Greifswald,

Ellernholzstrasse I-2, 17487

Greifswald, Germany

Tel +493834867 77I

Fax +493834867 752

Email neeltje.vandenberg@uni-greifswald.de 
indirect measurement enables the acquisition of subjects' personal perception of PA and a subjective estimate of intensity.

In many studies, the adherence of the participants wearing accelerometers according to the defined wearing scheme and suitable motivational strategies to increase adherence are not or only insufficiently reported..$^{8,9}$ However, the participants' adherence to the applied wearing scheme is a prerequisite for interpretable and valid study results.

We conducted a randomized controlled prospective study including 255 apparently healthy participants between 40 and 75 years of age who were recruited in different settings. Our goal was to explore which wearing scheme leads to the best adherence of wearing an accelerometer and to evaluate the potential additional effects of supportive phone calls (SPCs).

\section{Methods}

\section{Study design}

This study was part of a health examination program of participants aged 40-75 years from the German Center for Cardiovascular Research (DZHK).

Participants were recruited in three different settings: in general medical practices, in job agencies, and by invitation of a statutory health insurance. The participants recruited in general medical practices and job agencies were asked to complete a standardized health questionnaire to assess cardiovascular risk factors and lifestyle parameters, to undergo a blood pressure measurement, and to provide a blood sample directly in the recruitment setting. Participants selected and invited by the statutory health insurance were invited to an office in the examination center and followed the same procedure.

Subjects with a history of cardiovascular events (myocardial infarction and stroke), previous vascular interventions (open bypass surgery or percutaneous intervention), diabetes mellitus, multiresistant pathogens, or a self-reported body mass index (BMI) $>35 \mathrm{~kg} / \mathrm{m}^{2}$ were excluded from the study. Subjects without these exclusion criteria were invited for a subsequent cardiovascular examination program at the DZHK examination center.

A more detailed description of recruitment and the examination program was published in a previous study. ${ }^{10}$

\section{Accelerometer wearing schemes}

The subjects who participated in the health examination program and were examined in the examination center were invited to wear a three-axis accelerometer (GT3X+, Actigraph; Pensacola, FL, USA), fixed with an elastic band at the right hip. The subjects were asked to wear the accelerometer during
7 consecutive days "day and night" for 24 hours a day or "day only" after getting up in the morning and to put it off before going to sleep. All participants were instructed to remove the device only during water-based activities such as showering and bathing. The subjects were promised an individual feedback after wearing the accelerometer device.

Furthermore, all participants were asked to keep a semistandardized diary to document their PA frequency, duration, intensity, and all kinds of PA lasting for at least 15 minutes.

The duration of 7 consecutive days was chosen to include both weekdays and the weekends to examine both workrelated and leisure time activities ${ }^{7}$ and to reduce intraindividual variance.

\section{SPCs}

The SPCs consisted of two phone calls in which a member of the study team asked whether the participant have worn the accelerometer according to the instructions, whether the diary was kept, and to report any problems. The phone call was performed on the second and fourth days of the wearing period.

\section{Randomization}

The participants were at random assigned to the following four groups (Table 1):

- Group D: participants were instructed to wear the accelerometer according to the wearing scheme day only.

- Group DN: participants were instructed to wear the accelerometer according to the wearing scheme day and night.

- Group D + SPC: participants were instructed to wear the accelerometer during daytime. Additionally, this group received SPCs on the second and fourth days.

- Group DN + SPC: participants were instructed to wear the accelerometer day and night. Additionally, this group received SPCs on the second and fourth days.

\section{Participants}

Overall 492 individuals were invited to take part in the accelerometer adherence study, thereof 255 (51.8\%) gave informed consent and were randomized into one of the four groups D, DN, D+ SPC, and DN + SPC. The primary analysis was conducted with the data of 249 participants (Figure 1).

Table I Randomization groups as a two-factorial design

\begin{tabular}{lll}
\hline & Factor SPC \\
\hline Factor wearing time & D & $\mathrm{D}+\mathrm{SPC}$ \\
& $\mathrm{DN}$ & $\mathrm{DN}+\mathrm{SPC}$ \\
\hline
\end{tabular}

Abbreviations: D, day only; DN, day and night; SPC, supportive phone calls. 


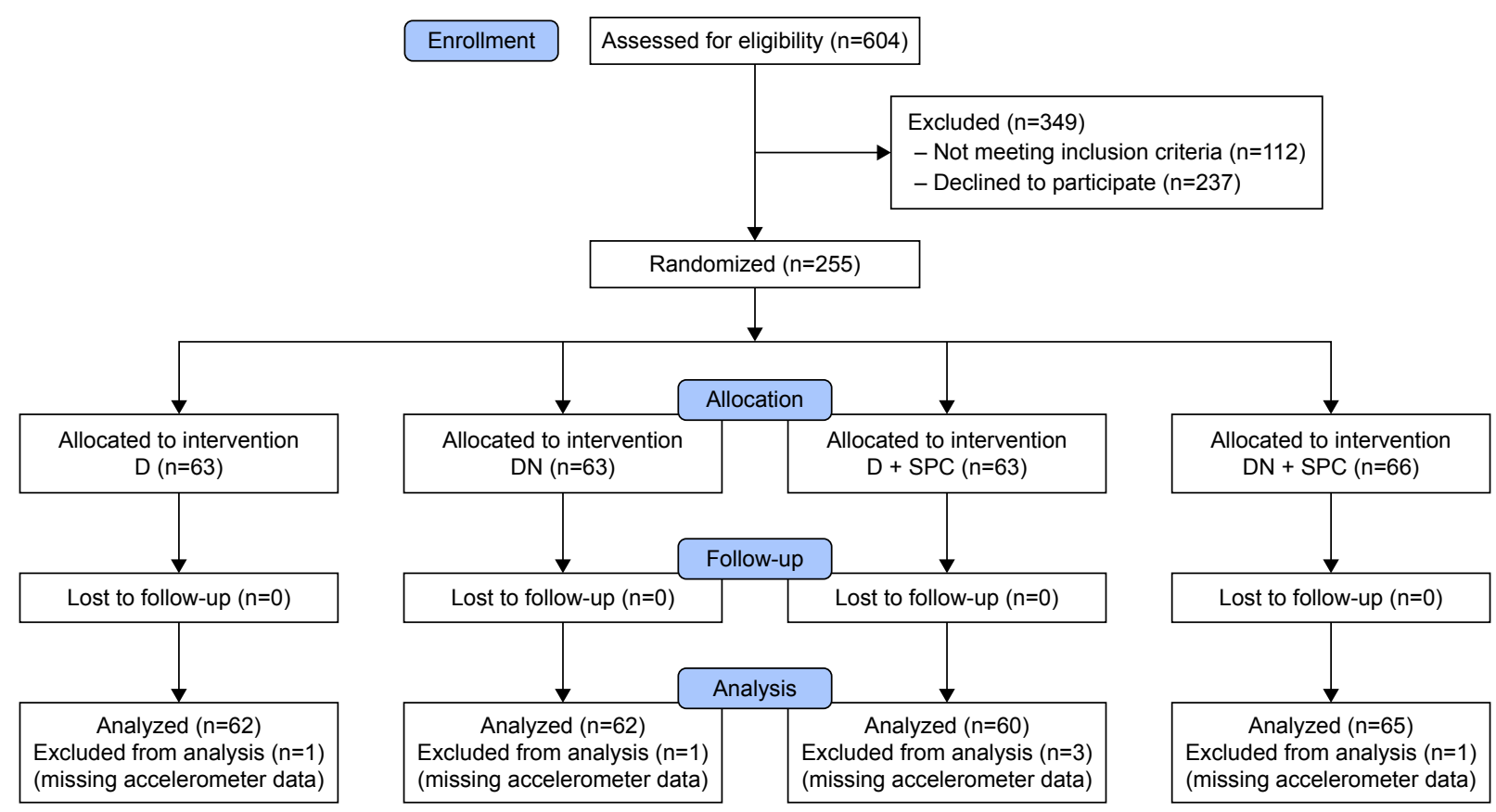

Figure I Consort flowchart.

Abbreviations: D, day; DN, day and night; D + SPC, day with supportive phone calls; DN+ SPC, day and night with supportive phone calls.

\section{Data analysis}

To compare the adherence between the different wearing schemes, the wearing time of the accelerometer between 8 am and $10 \mathrm{pm}$ during 7 consecutive days was assessed. This wearing time was chosen on the basis of typical wake up and bed times of the included population, assessed, and documented in a subsample $(\mathrm{N}=127)$ with completed diaries. $75 \%$ of the sample had to be awake to consider a time as wake up or bed time, respectively.

Full adherence was defined as a total wearing time of 98 hours ( 14 hours/d) between 8 am and $10 \mathrm{pm}$ during 7 days. Nonwear time was calculated by the Troiano algorithm (at least 60 consecutive minutes of zero activity intensity counts, with allowance for 1-2 minutes of counts between 0 and 100). ${ }^{11}$

Outcome parameter of the primary analysis was the difference between 98 hours and the observed wearing time. The distribution of this difference was highly right skewed.

Therefore, a generalized linear regression model on the basis of a negative binomial distribution was used for data analysis, whereas the effect estimator is the "incidence rate ratio" (IRR). Due to the difference to the maximum wearing time as the outcome variable, a lower IRR represents a higher adherence. The model included the two factors wearing scheme (DN or D) and SPC (Table 1).

The regression was performed as an intention-to-treat analysis. The interaction between the wearing time and SPC (yes or no) was used to test either multiplicative or additive interaction with the "relative excess risk due to interaction" (RERI). ${ }^{12}$

Additional to the primary analysis, two sensitivity analyses were performed: in the first sensitivity analysis, individual day-night structures were considered for 124 subjects with sufficient documentation of wake up and bed times in their diaries.

To examine a possible dose-effect relationship for the SPCs, a second sensitivity analysis was conducted for all participants in the two SPC groups taking the number of successful calls per protocol into account.

All analyses were computed with the software $\mathrm{R}$ version 3.1.1. For the negative binomial regression models, the MASS package was used (The R Foundation for Statistical Computing, Vienna, Austria).

\section{Ethics approval and consent to participate}

All participants gave written informed consent for this study. All participants received a voucher about 15 Euros upon return of the accelerometer after 7 days. The study was approved by the clinical ethical committee of the University Medicine Greifswald (protocol number BB64/07).

\section{Results}

The participants ( $40.0 \%$ men) were on average $56.4 \pm 9.8$ years old. Most subjects were participants from the setting "statutory health insurance" $(\mathrm{N}=120,47.1 \%)$. Further baseline characteristics are shown in Table 2. 
Table 2 Baseline characteristics by group

\begin{tabular}{|c|c|c|c|c|c|}
\hline & $D, n=63$ & $D N, n=63$ & $D+S P C, n=63$ & DN + SPC, n=66 & Total, $\mathbf{N}=\mathbf{2 5 5}$ \\
\hline Age (years), mean $\pm S D$ & $57.44 \pm 9.83$ & $57.38 \pm 10.01$ & $55.08 \pm 8.85$ & $55.65 \pm 10.33$ & $56.38 \pm 9.78$ \\
\hline Sex (\%), male & $21(33.3)$ & $31(49.2)$ & $25(39.7)$ & $25(37.9)$ & $102(40.0)$ \\
\hline \multicolumn{6}{|l|}{ Education (\%) } \\
\hline \multicolumn{6}{|l|}{ No or graduation } \\
\hline$<10$ years & $8(12.7)$ & II (I7.5) & $7(I I .1)$ & $12(18.2)$ & $38(14.9)$ \\
\hline 10 years & $29(46.0)$ & $25(39.7)$ & $33(52.4)$ & $34(51.5)$ & $|2|(47.5)$ \\
\hline$>10$ years & $25(39.7)$ & $26(41.3)$ & $23(36.5)$ & $20(30.3)$ & $94(36.9)$ \\
\hline Other graduation & $\mathrm{I}(\mathrm{I} .6)$ & $\mathrm{I}(\mathrm{I} .6)$ & $0(0)$ & $0(0)$ & $2(0.8)$ \\
\hline Living situation (\%), single household & $20(31.7)$ & $16(25.4)$ & $18(28.6)$ & $15(22.7)$ & $69(27.1)$ \\
\hline Smoking (\%), smoker & $18(28.6)$ & II (I7.5) & $17(27.0)$ & $15(22.7)$ & $61(23.9)$ \\
\hline \multicolumn{6}{|l|}{ Self-reported body mass index $\left(\mathrm{kg} / \mathrm{m}^{2}\right)$} \\
\hline Range (min-max) & |9.89-37.|| & $20.10-35.60$ & $18.44-35.92$ & $18.02-36.70$ & $|8.02-37.1|$ \\
\hline$\leq 25$ & $20(3 \mid .7)$ & $16(25.4)$ & $25(39.7)$ & $24(36.4)$ & $85(33.3)$ \\
\hline $25-30$ & $30(47.6)$ & $28(44.4)$ & $22(34.9)$ & $25(37.9)$ & $105(4 I .7)$ \\
\hline$>30$ & $13(20.6)$ & $19(30.2)$ & $16(25.4)$ & $17(25.8)$ & $65(25.5)$ \\
\hline \multicolumn{6}{|l|}{ Recruitment setting (\%) } \\
\hline Job agencies & $15(23.8)$ & $15(23.8)$ & II (I7.5) & II (I6.7) & $52(20.4)$ \\
\hline General medical practices & $17(27.0)$ & $19(30.2)$ & $25(39.7)$ & $22(33.3)$ & $83(32.5)$ \\
\hline Statutory health insurance & $31(49.2)$ & $29(46.0)$ & $27(42.9)$ & $33(50.0)$ & $120(47.1)$ \\
\hline
\end{tabular}

Abbreviations: D, day only; DN, day and night; D + SPC, day with supportive phone calls; DN + SPC, day and night with supportive phone calls; SD, standard deviation.

\section{Adherence, descriptive results}

The measure of adherence was assessed for 249 participants. For all four groups, the adherence was rather high (mean \pm standard deviation [SD] 84.04 \pm 20.75 hours). The adherence was better for the participants with the wearing scheme DN. Adding SPCs (on the second and fourth wearing days) tended to improve adherence further (97.56 hours without and 99.33 hours with SPCs), but the regression showed no significant enhancement in adherence (Table 3 ).

\section{Adherence, effects of wearing scheme and SPCs}

A negative binomial regression analysis was calculated for the difference between the maximal possible adherence (98 hours) and the observed adherence, including the independent variables wearing scheme (DN or D) and SPC (yes or no). A lower IRR represents a higher adherence.

Participants with the wearing scheme DN were more adherent than participants with the wearing scheme D (IRR
0.63, Table 4). SPCs had no significant influence on adherence. The negative binomial regression model including the multiplicative interaction was not significant (IRR 1.13, 95\% confidence interval [CI] 0.59-2.17, $P=0.712$, Table 4).

The additive interaction was analyzed regarding the randomization groups with wearing scheme $\mathrm{D}$ as the reference group. The RERI was positive, which represents a tendency to a positive additive interaction, but it was not significant (RERI $=0.16,95 \% \mathrm{CI}-0.28-0.61$, results not shown here).

\section{Results of the sensitivity analyses}

Table 5 shows the results of the sensitivity analysis on the basis of the individual wake up and bed time documentation of the participants. Wearing scheme and SPCs had no significant influence on adherence in this analysis.

The results of the subgroup analysis with participants of the groups with SPC on the second and fourth wearing days (with full adherence defined as a wearing time from 8 am until $10 \mathrm{pm}$ ) are shown in Table 6.

Table 3 Descriptive statistics of wearing time between 8 am and 10 pm (in hours) by group; N=249

\begin{tabular}{llllll}
\hline & $\mathbf{D}, \mathbf{n}=\mathbf{6 2}$ & $\mathbf{D N}, \mathbf{n}=\mathbf{6 2}$ & $\mathbf{D}+\mathbf{S P C}, \mathbf{n}=\mathbf{6 0}$ & $\mathbf{D N}+\mathbf{S P C}, \mathbf{n}=\mathbf{6 5}$ & Total, N=249 \\
\hline Mean \pm SD & $78.22 \pm 25.02$ & $86.34 \pm 19.57$ & $83.22 \pm 20.88$ & $88.15 \pm 15.78$ & $84.04 \pm 20.75$ \\
Median & $86.8 \mathrm{I}$ & 93.94 & 89.76 & 93.82 & 91.47 \\
Range (min-max) & $00.00-97.96$ & $9.64-98.00$ & $0.01-98.00$ & $00.00-98.00$ & $00.00-98.00$ \\
IQR (x0.25-x0.75) & $73.83-94.31$ & $86.66-96.61$ & $81.80-93.44$ & $86.79-95.88$ & $82.53-95.37$ \\
Difference to 98 h, (mean \pm SD) & $19.78 \pm 25.02$ & $11.66 \pm 19.57$ & $14.78 \pm 20.88$ & $9.85 \pm \mid 5.78$ & $13.96 \pm 20.75$ \\
\hline
\end{tabular}

Abbreviations: D, day only; DN, day and night; D + SPC, day with supportive phone calls; DN + SPC, day and night with supportive phone calls; h, hours; IQR, interquartile range; SD, standard deviation. 
Table 4 Results of the primary regression model by a negative binomial regression approach

\begin{tabular}{|c|c|c|c|c|c|c|}
\hline \multirow{2}{*}{$\begin{array}{l}8 \mathrm{am} \text { to } 10 \mathrm{pm} \\
(\mathrm{N}=249)\end{array}$} & \multicolumn{3}{|c|}{ Model } & \multicolumn{3}{|c|}{ With interaction } \\
\hline & IRR & $95 \% \mathrm{Cl}$ & $P$-value & IRR & $95 \% \mathrm{Cl}$ & $P$-value \\
\hline $\begin{array}{l}\text { Wearing time } \\
\text { (DN vs day only) }\end{array}$ & 0.63 & $0.45-0.87$ & 0.005 & 0.59 & $0.37-0.94$ & 0.024 \\
\hline SPC vs none & 0.80 & $0.57-1.10$ & 0.168 & 0.75 & $0.47-1.19$ & 0.219 \\
\hline $\begin{array}{l}\text { Interaction } \\
\text { (DN by SPC) }\end{array}$ & - & - & - & 1.13 & & 0.712 \\
\hline
\end{tabular}

Note: Dependent variable: difference between the maximal possible adherence (98 hours for 7 consecutive days) and the observed adherence (based on wearing time from 8 am until $10 \mathrm{pm}$ ).

Abbreviations: $\mathrm{Cl}$, confidence interval; $\mathrm{DN}$, day and night; IRR, incidence rate ratio; SPC, supportive phone calls.

The two groups of participants with SPC had in total $\mathrm{N}=127$ participants, thereof $\mathrm{n}=63(49.6 \%)$ received the intended two calls, $\mathrm{n}=47(37.0 \%)$ received one call, and $\mathrm{n}=17$ (13.4\%) participants received no call at all. Participants who received SPC showed better adherence compared to participants who did not receive these calls, but this effect did not reach statistical significance. The number of SPCs had no influence on the adherence.

\section{Discussion}

The results of this study document a high adherence to wearing an accelerometer across all groups during the predefined time range. The descriptive results showed a tendency to better adherence for the group with the wearing scheme DN than the wearing scheme D. Additional SPC did not increase adherence in both groups.

In general, we observed a high mean adherence across all groups. This may be a consequence of the selection of the participants: this study was part of a survey among healthy persons including questionnaires and examinations without a subsequent intervention. It is a plausible assumption that the participants are not representative compared to the general population. A comparison of the participant sample with the

Table 5 Results of the subgroup analysis for participants with completed diary data of the four groups, regarding the relative adherence by a negative binomial regression approach

\begin{tabular}{llll}
\hline $\mathbf{8}$ am to $10 \mathbf{~ p m ~ ( N = I 2 4 )}$ & IRR & $\mathbf{9 5 \%} \mathbf{C l}$ & $\mathbf{P}$-value \\
\hline Age (years) & I.0I & $0.95-I .08$ & 0.680 \\
Sex (vs female) & 0.88 & $0.25-2.8 I$ & 0.836 \\
Wearing scheme (DN vs day only) & 0.50 & $0.12-1.65$ & 0.282 \\
SPC vs none & 0.98 & $0.31-3.34$ & 0.975 \\
\hline
\end{tabular}

Note: Dependent variable: difference between the maximal possible adherence (98 hours for 7 consecutive days) and the observed adherence (based on wearing time from 8 am until $10 \mathrm{pm}$ ).

Abbreviations: $\mathrm{Cl}$, confidence interval; $\mathrm{DN}$, day and night; IRR, incidence rate ratio; SPC, supportive phone calls.
Table 6 Subgroup analysis considering the number of supportive phone calls, adjusted for age and sex

\begin{tabular}{llll}
\hline $\mathbf{8}$ am to $10 \mathbf{~ p m ~ ( N = 1 2 7 )}$ & IRR & $\mathbf{9 5 \%} \mathbf{C l}$ & $\mathbf{P}$-value \\
\hline Age (years) & 1.02 & $1.00-1.04$ & 0.163 \\
Sex (vs female) & 0.66 & $0.41-1.06$ & 0.055 \\
Wearing time (DN vs day only) & 0.60 & $0.38-0.95$ & 0.018 \\
SPC vs no call & & & \\
$\quad$ One phone call & 0.59 & $0.28-1.19$ & 0.126 \\
$\quad$ Two phone calls & 0.62 & $0.30-1.18$ & 0.145 \\
\hline
\end{tabular}

Note: Dependent variable: difference between the maximal possible adherence (98 hours for 7 consecutive days) and the observed adherence (based on wearing time from 8 am until $10 \mathrm{pm}$ ).

Abbreviations: $\mathrm{Cl}$, confidence interval; $\mathrm{DN}$, day and night; IRR, incidence rate ratio; SPC, supportive phone calls.

general population (based on data of a large German survey) showed differences with respect to some important parameters: $27.1 \%$ vs $40.1 \%$ living in a single-person household and $23.9 \%$ vs $24.5 \%$ smokers. ${ }^{13,14}$

The sensitivity analysis including the subgroup with known day-night patterns showed less difference between the wearing schemes than the primary analysis. This could be an indication that the difference in the primary analysis could partly be the consequence of generalizing individual day-night patterns. However, this subgroup was selected because their diaries were precise enough to determine individual patterns. This accuracy could have an influence also on the adherence to the wearing scheme.

Additional SPCs did not add significantly to an increased adherence in this study. Conceivably, due to the selected, probably highly motivated group of participants, a ceiling effect may have occurred. Hence, motivational calls could improve adherence in less selected and motivated groups. In a study among 60 economically disadvantaged women in South Carolina, USA, three reminder telephone calls were implemented to optimize adherence to wearing an accelerometer from wake up until bedtime during a time period of 7 days. In this study, wearing the accelerometer at least 4 days for at least 10 hours a day was defined as adherent, and 57 of 60 women $(95 \%)$ reached adherence. ${ }^{15}$

In a study including 89 high school students in Minneapolis, USA, the participants were assigned to one of three compliance interventions (receiving three telephone contacts, completing a daily journal, and receiving compensation dependent on adherence) or to a control group. The students receiving adherence-dependent compensation showed the highest adherence ( $96 \%$ of the participants in this group completed at least 4 of 7 days with a daily wearing time of at least 10 hours). The other interventions did not show significantly higher adherence rates than the control group. ${ }^{16}$ 
In a sample of 117 adolescents in Ireland, to wear the accelerometer over daytime was more likely if the students received an SMS reminder message each morning. ${ }^{17}$ The results of these studies indicate a positive influence of motivational support on adherence. However, a comparison to our study is limited because of different population groups.

Our study has some limitations. First, the study sample was selected with respect to the recruitment settings and health status. It is likely that this sample is not representative for the general German population $\geq 40$ years. The results may therefore not readily extend to other groups or Germany population as a whole.

In this study, we aimed to assess adherence wearing the accelerometer during the day. We found that the definition of "daytime" is a challenge. However, including individual day-night patterns requires an accurate documentation by the participants, which is an elaborate task and prone to missing or inaccurate entries. Determining daytime for the whole group leads to a generalized reflection of real life, which may have biased the results to some extent. Since we found better adherence for the wearing scheme day and night, it might be a solution in some cases to count total PA ( 24 hours) to avoid this problem.

\section{Conclusion}

The results of this study show a better adherence for wearing the accelerometer in subjects with a wearing scheme day and night. Additional SPCs did not improve adherence further.

These results are a good basis for further research with respect to adherence. Especially, whether SPCs or other interventions (eg, supportive SMS) may have an additional value in terms of increased adherence in selected target populations needs further evaluation.

\section{Acknowledgments}

This work was supported by the German Federal Ministry of Education and Research (grant number 81Z7400174). This study is registered at the German Clinical Trials Register (https://drks-neu.uniklinik-freiburg.de/drks web/; no DRKS00010996).

\section{Disclosure}

The authors report no conflicts of interest in this work.

\section{References}

1. Westerterp KR. Physical activity assessment with accelerometers. Int J Obes Relat Metab Disord. 1999;23(suppl 3):S45-S49.

2. Johnsen NF, Ekblond A, Thomsen BL, Overvad K, Tjønneland A. Leisure time physical activity and mortality. Epidemiology. 2013;24: 717-725.

3. Carlsson AC, Ärnlöv J, Sundström J, Michaëlsson K, Byberg L, Lind L. Physical activity, obesity and risk of cardiovascular disease in middleaged men during a median of 30 years of follow-up. Eur J Prev Cardiol. 2016;23(4):359-365.

4. Li J, Siegrist J. Physical activity and risk of cardiovascular disease a meta-analysis of prospective cohort studies. Int J Environ Res Public Health. 2012;9(2):391-407.

5. Naci H, Ioannidis JP. Comparative effectiveness of exercise and drug interventions on mortality outcomes: metaepidemiological study. $B M J$. 2013;347:f5577.

6. Fiuza-Luces C, Garatachea N, Berger NA, Lucia A. Exercise is the real polypill. Physiology (Bethesda). 2013;28(5):330-358.

7. Strath SJ, Kaminsky LA, Ainsworth BE, et al. Guide to the assessment of physical activity: clinical and research applications: a scientific statement from the American Heart Association. Circulation. 2013; 128(20):2259-2279.

8. Harris TJ, Owen CG, Victor CR, Adams R, Cook DG. What factors are associated with physical activity in older people, assessed objectively by accelerometry? Br J Sports Med. 2009;43(6):442-450.

9. Murphy SL. Review of physical activity measurement using accelerometers in older adults: considerations for research design and conduct. Prev Med. 2009;48(2):108-114.

10. Weymar F, Braatz J, Guertler D, et al. Characteristics associated with nonparticipation in 7-day accelerometry. Prev Med Rep. 2015;2:413-418.

11. Troiano RP, Berrigan D, Dodd KW, Mâsse LC, Tilert T, McDowell M. Physical activity in the United States measured by accelerometer. Med Sci Sports Exerc. 2008;40(6):181-188.

12. Hosmer DW, Lemeshow S. Confidence interval estimation of interaction. Epidemiology. 1992;3(5):452-456.

13. Statistisches Bundesamt. Haushalte und Familien. Available from: https://www.destatis.de/DE/ZahlenFakten/GesellschaftStaat/ Bevoelkerung/HaushalteFamilien/HaushalteFamilien.html. Accessed August 19, 2015.

14. Statistisches Bundesamt [webpage on the Internet]. Neues Jahr, alte Vorsätze: Fakten zum Rauchen. Available from: https://www.destatis. de/DE/ZahlenFakten/ImFokus/Gesundheit/Rauchen.html. Accessed August 19, 2015.

15. Sharpe PA, Wilcox S, Rooney LJ, et al. Adherence to accelerometer protocols among women from economically disadvantaged neighborhoods. J Phys Act Health. 2011;8(5):699-706.

16. Sirard JR, Slater ME. Compliance with wearing physical activity accelerometers in high school students. J Phys Act Health. 2009; 6(suppl 1):S148-S155.

17. Belton S, O'Brien W, Wickel EE, Issartel J. Patterns of noncompliance in adolescent field-based accelerometer research. J Phys Act Health. 2013; 10(8):1181-1185.
Patient Preference and Adherence

\section{Publish your work in this journal}

Patient Preference and Adherence is an international, peer-reviewed, open access journal that focuses on the growing importance of patient preference and adherence throughout the therapeutic continuum. Patient satisfaction, acceptability, quality of life, compliance, persistence and their role in developing new therapeutic modalities and compounds to optimize

\section{Dovepress}

clinical outcomes for existing disease states are major areas of interest for the journal. This journal has been accepted for indexing on PubMed Central. The manuscript management system is completely online and includes a very quick and fair peer-review system, which is all easy to use. Visit http://www. dovepress.com/testimonials.php to read real quotes from published authors. 